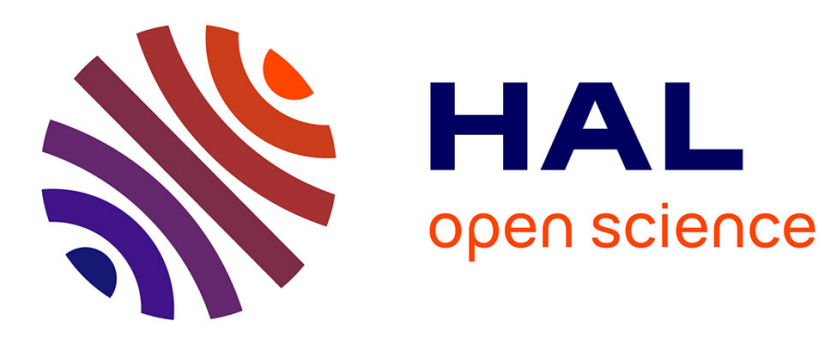

\title{
Le concept heuristique d'affections psychosomatiques du cerveau
}

\author{
Georgios (Yorgos) Dimitriadis
}

\section{To cite this version:}

Georgios (Yorgos) Dimitriadis. Le concept heuristique d'affections psychosomatiques du cerveau. L'Évolution Psychiatrique, 2013, 78 (2), pp.290-300. 10.1016/j.evopsy.2012.09.007 . hal-01467030

\section{HAL Id: hal-01467030 \\ https://hal.science/hal-01467030}

Submitted on 14 Feb 2017

HAL is a multi-disciplinary open access archive for the deposit and dissemination of scientific research documents, whether they are published or not. The documents may come from teaching and research institutions in France or abroad, or from public or private research centers.
L'archive ouverte pluridisciplinaire HAL, est destinée au dépôt et à la diffusion de documents scientifiques de niveau recherche, publiés ou non, émanant des établissements d'enseignement et de recherche français ou étrangers, des laboratoires publics ou privés. 


\section{Le concept heuristique d'affections psychosomatiques du cerveau}

\section{Yorgos Dimitriadis}

Lacan était un homme de son temps, partie prenante des débats de son époque, ce qui demeure un enseignement pour le psychanalyste d'aujourd'hui.

Alain Vanier [1]

\section{Sommes-nous notre cerveau?}

L'idée que nous sommes notre cerveau, c'est à dire que le sujet que nous sommes est, en fait, cette partie du corps qu'on appelle l'encéphale, est très répandue de nos jours. Le fondement de cette idée commence probablement avec l'histoire de l'individualisme possessif selon lequel on est le propriétaire de notre personne et sans dette vis-à-vis de la société. Selon Fernando Vidal [2] cette idée est une suite logique de la philosophie de l'identité et de la matière du $17^{\text {ème }}$ : à savoir que de l'idée de John Locke que notre identité est individuelle et relative à notre conscience et notre mémoire on est passé à celle de Charles Bonnet selon laquelle c'est notre cerveau qui définit notre identité. Cette cérébralisation progressive de l'identité qu' Alain Ehrenberg a soutenu également [3] a sans doute aussi propulsé les recherches neuroscientifiques. Mais les découvertes des neurosciences durant ces dernières décennies ont voulu légitimer, en retour, cette idée de la cérébralité du sujet. Les recherches neuroscientifiques ont mis en évidence, entre autres, des changements des structures cérébrales et des modifications de neurotransmetteurs dans les maladies psychiatriques. L'idée que nous sommes notre cerveau a donné des arguments pour que la neurologie et la psychiatrie se retrouvent à 
nouveau en situation de noces après un intervalle de séparation mais sans véritable divorce $^{1}$. D'un autre côté cette même idée a limité les affections psychosomatiques au soma périphérique. Car si on identifie notre psyché - et nous même - à notre cerveau il ne reste que le soma périphérique pour souffrir d'une telle affection. Comme le dit pourtant Stéphane Thibierge [6] le fonctionnement du cerveau dépend de la structure et de la physiologie des neurones, tandis que le fonctionnement psychique dépend des structures langagières, symboliques et extérieures aux cerveaux individuels. Quand Henri Ey [7] rédigeait l'acte de séparation entre la neurologie et la psychiatrie, il laissait la porte ouverte aux retrouvailles entre elles lorsque, par exemple, avec sa théorie sur la conscience, les démences restaient dans le champ de la psychiatrie. La voix de Jacques Lacan [8] à Bonneval avec son discours sur la causalité psychique et même après [9], une dizaine d'années plus tard, quand il disait que la seule organicité en rapport avec la psychose est celle qui motive la structure de la signification, n'a pas pu faire objection définitive à cette « conscience malade » qu' Ey avait pu considérer comme étant à la base de certaines maladies mentales.

\section{Psychosomatique et cerveau}

Pendant les années '60, c'est-à-dire au moment du début des découvertes neuroscientifiques, certains avaient pu penser que le cerveau pourrait être atteint d'un processus psychosomatique : par exemple le

\footnotetext{
${ }^{1}$ Cf. par exemple Price, Adams \& Coyle [4] et Yodofsky \& Hales [5]
} 
psychiatre et psychanalyste américain Silvano Arieti [10] avait avancé que la schizophrénie, et secondairement la psychose maniacodépressive, pourraient être considérées comme des maladies mentales où il y a une participation psychosomatique du cerveau. Idée qui existait déjà implicitement chez Carl Gustave Jung [11] quand il avançait une hypothèse endotoxinique pour la schizophrénie. Car, selon Jung, il existe au départ de cette maladie un complexe idéo-affectif qui produit un affect fort à partir duquel les troubles de l'humeur initiaux se développent, lesquelles, par l'intermédiaire d'un facteur métabolique $\mathrm{X}$, fixent et automatisent les complexes idéo-affectifs comme d'autres phénomènes psychiques à tonalité surtout affective. Cette idée a été reprise par Arieti [12] dans son hypothèse, cette fois-ci, explicitement psychosomatique sur la schizophrénie. Arieti y voyait une intégration des fonctions cérébrales les plus élevées à un niveau moins élevé. Ainsi selon Arieti [11] le raisonnement paléologique, les perceptions et le présent viennent à la surface à la place d'un symbolisme interpersonnel plus complexe. Depuis d'autres auteurs comme Dejours et Abdoucheli [13], Laznik [14], Mentzos [15], Pally [16], et Widlocher [17] ont évoqué explicitement la possibilité d'une «psychosomatique du cerveau» et d'autres auteurs encore comme Ali [18], Hartocolis [19], et Verhaeghe [20] ont souligné les rapports de la psychose avec les affections psychosomatiques.

Mais comment les théories psychosomatiques plus récentes peuvent elles nous éclairer sur ces états? Nous avons préféré la théorie lacanienne aux autres théories car elle va nous permettre de nouer notre argument avec la sémiotique et d'introduire le concept de réduction sémiotique qui 
est le fil rouge de notre argument. Venons aux prémisses de ce que Lacan a développé sur la question de la psychosomatique. A notre avis le moment le plus fort dans son enseignement à ce propos est l'idée d'une "gélification de la chaîne signifiante », que Lacan [21,22] évoquait comme susceptible d'avoir un rapport avec les phénomènes psychosomatiques (mais aussi avec d'autres états cliniques comme la paranoïa et l'arriération mentale). Selon Lacan les signifiants ne renvoient pas, à travers une opération binaire, à des choses (comme c'est le cas pour les signes) mais à d'autres signifiants à travers la dialectique du désir de chaque sujet qui est accroché au désir de l'Autre. Il s'agit donc d'opérations ternaires. L'exemple princeps, par rapport à cette dialectique, est l'infans qui, en tant que sujet de désir, a affaire avec sa mère (ou le prochain secourable en général) et, à travers elle, avec un tiers terme qui est - par exemple - le désir de la mère pour le père.

Selon Lacan quand il y a une gélification de la chaîne signifiante la dialectique du désir s'arrête et « le signifiant du désir de l'Autre »- de ce fait - obtient une opacité, il devient mystérieux. A cet état il arrête de renvoyer à un autre signifiant pour devenir un inducteur, un signal, qui induit des perturbations aux besoins du soma au lieu de relancer la dialectique du désir du sujet. Lacan a mis explicitement la théorie de Pavlov sur le conditionnement en rapport avec son hypothèse de gélification de la chaîne signifiante dans les phénomènes psychosomatiques. C'est à dire que selon Lacan il y aurait une analogie entre le signifiant gelé et le signal de l'expérimentateur de l'expérience de Pavlov (de la sonnette à la place de la viande) quand il essayait de 
conditionner l'animal domestiqué (et comme tel sensible aux signes venant de l'autre humain), à savoir le chien. Cette théorisation permet de mettre en rapport la théorie psychanalytique de Lacan sur la psychosomatique, primo, avec la sémiotique et, secundo, avec la neurophysiologie. Nous avons appelé [23] cette réduction du signifiant au signal, voire même aux stimuli, «processus de réduction sémiotique ». Comme nous venons de le dire, dans ce type de processus le signal ainsi produit obtient une « capacité » impérative pour le sujet et conditionne son soma et il peut, par cette voie, provoquer une perturbation des fonctions, voire des lésions. En parlant de besoins nous ne faisons pas référence seulement à la faim ou au besoin d'exonération. Il s'agit en fait de plusieurs circuits homéostatiques de l'organisme qui peuvent être dérangés par le désir et les pulsions. Sur ce point ce que nous croyons apporter de nouveau est la généralisation de cette théorie lacanienne en postulant qu'il y a la possibilité d'une atteinte, par ce type de processus sémiotique, également des circuits homéostatiques du cerveau. C'est-à-dire qu' à notre avis le signal peut conditionner, en dehors des circuits homéostatiques du soma périphérique, aussi les circuits du cerveau. Comme nous verrons plus loin les circuits qui régulent nos humeurs seraient peut être à cet égard une cible privilégiée.

\section{Le processus de réduction sémiotique et la Phanéroscopie}

Mais avant de poursuivre sur les circuits cérébraux qui régulent l'humeur, restons un instant sur ce qui se passe au niveau de cette réduction du signifiant au signal ou aux stimuli. Nous pensons que ce processus peut être explicité par le biais de la Phanéroscopie (la théorie 
des catégories de la phénoménologie) de Peirce [24] avec ses trois catégories : la priméité, la secondéité et la tiercéité. La priméité est le « mode d'être de ce qui est tel qu'il est, positivement et sans référence à quoi que ce soit d'autre ». La sécondéité est le «mode d'être de ce qu'il est tel qu'il est, par rapport à un second, mais sans considération d'un troisième quel qu'il soit ». La tiercéité est le « mode d'être de ce qui est tel qu'il est, en mettant en relation réciproque un second et un troisième ». La priméité est en rapport avec le sentiment immédiat, la secondéité avec la réaction et l'actualité, et la tiercéité avec le langage, la loi et la représentation. La tiercéité serait la catégorie proprement humaine. La sémiotique de l'homme est régie par cette dialectique ternaire car, en dehors de la dialectique de son désir - dont nous avons parlé plus haut -, des processus tels que la comodalisation des différents flux sensoriels, l'attention partagée, les jeux de faire semblant, les dites métareprésentations, les « jeux langagiers », les structures de parenté etc. sont des processus ternaires. Pour les animaux c'est la secondéité des signaux qui régit leurs systèmes sémiotiques. Selon Jean-Marie Vidal [25] «ces répertoires de signaux, dont chacun est étroitement lié au stimulus qu'il signale, au point de fonctionner comme ce dernier, procèdent de systèmes de 'liens dyadiques' ou du principe de la 'secondéité', là où les stimuli eux -mêmes agissent seuls, en 'monades', selon le principe de la 'priméité' ». Pour les signaux le jeu de déplacement se limite à des rapports de présence synchronique (de contiguïté temporelle ou spatiale par exemple) et non pas à des rapports (diachroniques) de référence à quelque chose d'absent, comme les mots. La fonction du langage, disait Lacan [26] à 
propos, n'est pas d'informer mais d'évoquer. Les reflexes naturels ou conditionnés et, de manière plus générale, les réactions immédiates à un signal, seraient donc de l'ordre de la secondéité. Les stimuli n'ont même pas besoin d'un autre signe pour être efficaces, ils agissent par eux même en circuit fermé (en monades) et peuvent ainsi s'auto-entretenir. Vidal [25], a postulé que les patientes autistes désymbolisent ou détiercéisent les formes symboliques en leur substituant des formes dyadiques ${ }^{2}$. A l'aide de la Phanéroscopie de Peirce, nous proposons pour plusieurs psychopathologies un passage progressif de la tiercéité vers des états qui relèvent de la secondéité ou de la priméité. C'est à dire un passage vers des états de plus en plus automatiques.

Plus précisément :

- Virer vers la secondéité équivaudrait, sur le plan de la sémiotique, au passage du signifiant au signal et, sur le plan clinique, nous avons comme effet des phénomènes de conditionnement et des phénomènes réactionnels en général. Par exemple nous avons certains phénomènes psychosomatiques classiques, les crises d'angoisse conditionnées, certains phénomènes de conditionnement chez les toxicomanes, des dépressions ou manies réactionnelles, le syndrome de répétition dans la névrose traumatique, certains « symptômes » à caractère d'acte et autres.

- Le passage de la tiercéité ou de la secondéité vers la priméité irait même au-delà de ce «stade » de réduction. Nous pourrions dire, peut être, qu'on

\footnotetext{
${ }^{2}$ D'autres auteurs [27-30] ont aussi utilisé les catégories de la phanéroscopie de Peirce dans le champ de la psychopathologie.
} 
passe du signifiant ou du signal aux stimuli. Dans ce cas nous avons des états encore plus automatiques comme les troubles de l'humeur automatiques : par exemple humeur délirante stable ou athymhormie chez le schizophrène, états maniaques et dépressifs devenus autonomes par rapport à leurs causes déclenchantes initiales, états de panique automatiques, certains phénomènes psychosomatiques automatiques ou autres. Dans tous ces états les signes ne viennent pas de l'autre comme c'est le cas par exemple dans le conditionnement où il y a le signal de l'autre qui y joue. Ici d'une certaine manière, les stimuli s'autoentretiennent.

Bien évidemment avec cette éventualité de réduction sémiotique nous ne soutenons pas une continuité entre l'animal et l'homme. La tiercéité, même dans ces états limites, reste présente car elle est constitutive pour l'homme qui ne peut pas y faire dérogation. Les systèmes éthologiques de signalisation (signaux et stimuli) chez l'homme sont subjugués (et dénaturalisés d'une certaine manière) par les structures ternaires. D'ailleurs la preuve en est que quand la tiercéité est compromise, comme dans les états précédents, le corps humain se rend malade.

\section{Logique du signifiant versus logique du signe}

Mais en dehors de la Phanéroscopie de Peirce nous pouvons spécifier ce processus de réduction sémiotique par rapport aux notions psychanalytiques de «l'effet après coup» et de «répétition ». Le symbolique, le réseau de signifiants d'un sujet particulier, n'est pas un système clos. Chaque rencontre avec le hasard peut modifier la chaîne de ses signifiants. Chaque signifiant 
peut modifier l'ensemble de la chaîne signifiante d'un sujet. Dans le cas de la cure psychanalytique, l'isolement d'un signifiant (d'un point de capiton) peut permettre au sujet de donner une nouvelle signification rétroactive de toute son histoire. Cette conceptualisation est une lecture plus radicale du concept de «l'action après coup » des événements, de la Nachträglichkeit de Freud. Si nous considérons que pour tel sujet certains signifiants ont joué un rôle particulier, ceux-ci peuvent obtenir des significations différentes selon les périodes de sa vie mais n'arrêtent pas pour autant d'avoir une valeur déterminante pour ce sujet. Ce sont une limite, une castration symbolique, pour ce sujet particulier. Mais cette re-détermination récurrente, autour des éventualités que le symbolique impose au sujet, ouvre en même temps de nouvelles dimensions, à condition pourtant qu'il puisse «admettre » son inscription au symbolique, c'est à dire qu'il puisse accepter la limite de la castration que son histoire personnelle lui impose. Ainsi un sujet, en arrêtant de considérer que toutes les possibilités lui sont ouvertes (et la problématique de l'obsessionnel est particulièrement parlante à cet égard), permet à ses contingences d'apparaître (de s'inscrire) et transforme, par là même, ce qui lui est contingent en nécessité pour son avenir. Donc, la logique du signifiant est une logique qui «prépare » l'homme au hasard de la rencontre, à l'imprévisibilité de l'émergence contingente du désir de l'Autre, et à la singularité. Cette logique est diachronique et de rétroaction récurrente - selon laquelle le résultat peut agir sur sa cause et la modifier dans l'après coup ${ }^{3}$. Par contre la logique du signal ou du signe est une logique linéaire valable pour

\footnotetext{
${ }^{3}$ François Ansemet et Pierre Magistretti [31] ont écrit sur cette opération en rapport avec la neuroplasticité. D'autres auteurs comme Karen Ruth-Lyons [32] ont exprimés des vues semblables.
} 
les réflexes, naturels ou conditionnés, et implique une objectivation, une universalité de réactions. Elle implique aussi une synchronie et/ou une contiguiité spatiale et elle régit l'apprentissage chez les animaux.

Alors, nous pouvons envisager la « réduction sémiotique » également dans ses conséquences d'arrêt du «capitonnage » ${ }^{4}$ par la chaîne signifiante. Cet arrêt du capitonnage pourrait survenir dans des contextes variés et nous parlerons plus loin - à titre d'exemple - de ce qui peut se passer chez le schizophrène. Le capitonnage impose une assomption subjective et diachronique, car il met en liaison à travers les signifiants. Ce capitonnage est aussi une assomption de la contingence, de ce qui se produit de nouveau pour un sujet. Il est donc, selon l'expression de Georges Lanteri-Laura [34], un automatisme créateur. Par l'arrêt du capitonnage, la rencontre avec les signifiants du désir de l'Autre obtient une objectivité, et une actualité et cette actualité a un caractère d'injonction, un caractère signalétique. Ces signifiants gelés et « impératifs » sont des pseudo-signifiants «coupés » de l'histoire (de la diachronie du sujet). En fait ces sont des signaux qui peuvent déclencher des processus psychosomatiques. Voici donc une autre manière d'entendre le terme de «névroses actuelles ${ }^{5}$ car les signaux, a contrario des signifiants, agissent dans la synchronie et l'actualité. Plus spécifiquement dans les troubles de l'humeur, la réduction sémiotique (ou l'arrêt du capitonnage) consisterait en ceci : les affects, liés aux signifiants du sujet -

\footnotetext{
${ }^{4}$ Marcel Czermak [33] emploie le néologisme de décapitonnage à propos de la manie et l'adjectif 'désaffecté' pour le sujet maniaque et de 'semblants d'affects' pour les affects quand il n'y a plus de capitonnage par la chaine signifiante dans la manie

${ }^{5}$ Terme, comme on sait, prédécesseur du terme de maladies psychosomatiques. Charles Melman [35] avec son concept de «nouvelle économie psychique », inspire et prolonge cette lecture sur l'actualité
} 
quoique de manière déplacée - perdent ce lien, c'est-à-dire qu'ils se désaffectent de la fonction signifiante, et, par là même, se transforment en émotions (de l'ordre de la sécondéité) ou en humeur (de l'ordre de la priméité). Nous verrons par la suite cette idée appliquée plus précisément dans le cadre de la schizophrénie.

\section{Participation psychosomatique du cerveau dans la schizophrénie}

Pourrait-on donner une plausibilité neurophysiologique à notre argument? Mais pourquoi rechercher cette plausibilité qui n'est d'ailleurs pas essentielle pour notre argument psychanalytique. Nous pensons que cette plausibilité neurophysiologique indique une voie suivant laquelle nous pouvons distinguer la neurophysiologie ou même la neuropathologie retrouvées dans les maladies psychiatriques par rapport à celles qu'on retrouve dans des maladies neurologiques. Robert Post [36], par exemple, a indiqué comment le mécanisme neurophysiologique de l'embrasement (kindling) pourrait être appliqué dans la compréhension des troubles de l'humeur, et d'autres pathologies psychiatriques. En ce qui concerne les troubles de l'humeur il a postulé que la progression de la maladie maniacodépressive peut aller d'un mode de fonctionnement réactionnel vers un mode de fonctionnement automatique. Ceci à travers une évolution des épisodes dysthymiques qui deviennent d'abord réactionnels et conditionnés par rapport à certaines circonstances particulières. Puis, dans un deuxième temps, s'ils se répètent suffisamment fréquemment, ces épisodes dysthymiques peuvent devenir autonomes, c'est à dire automatiques. A savoir que l'automatisme dépend de l'état d'excitation qui tend à s'auto-entretenir, d'où d'ailleurs le terme 
d'embrasement. Stephan Stahl [37] soutient que cette excitation neuronale peut même devenir toxique et détruire certains neurones. Selon cet auteur, dans certaines situations cliniques comme la schizophrénie, la dépression, le trouble bipolaire, le trouble panique, la maladie d'Alzheimer, la maladie de Parkinson et d'autres, l'excitotoxicité provoque une apoptose neuronale et rend ces états irréversibles, au moins pour une part,. Nous voyons comment ce dernier auteur fait un amalgame entre maladies neurologiques et psychiatriques.

Si, néanmoins, nous prenons les choses à partir de ce que nous venons d'avancer comme processus de réduction sémiotique, la progression du réactionnel vers l'automatique pourrait être concevable pour certaines maladies psychiatriques comme un processus psychosomatique. Ici nous allons nous limiter à l'exemple de la schizophrénie. Dans d'autres articles [38-39] aussi nous avons évoqué comment la défaillance de la fonction signifiante par la forclusion du Nom-du-Père chez le schizophrène peut provoquer des perturbations de l'image de son corps et comment ces perturbations, de la fonction signifiante et de l'image de son corps, l'empêchent de se situer dans le contexte des événements. De ce fait le sujet est envahi par des signes car même des stimulations qu'il devrait ignorer deviennent saillantes pour lui. Plusieurs auteurs [40-47] ont soutenu une telle hypothèse et ce à partir de points de vues très différents. Lacan [48, p.137] dans son travail précoce de sa thèse, a qualifié cette phase d' " état affectif presque pur ». Il s'agit ici, selon nous, d'un passage de la tiercéité signifiante vers la secondéité de signes. A partir de ce point le sujet peut construire un délire organisé qui peut être, selon Freud [49], 
une voie de guérison. Mais s' il n'arrive pas par la voie du symbolique c'est à dire par des métaphores délirantes - ou par des compensations imaginaires et par d'autres types de suppléances ${ }^{6}$ à trouver une issue pacificatrice, l'état précédent - de l'envahissement par les signes - peut déclencher à son tour deux processus humoraux que nous avons considérés comme des processus psychosomatiques du cerveau. Là on passe de la secondéité des signes à la priméité de l'humeur. Le premier de ces deux processus serait celui d'une « humeur délirante stable», qui donne au sujet une tendance à chercher des coïncidences (c'est-à-dire une synchronie ou des ressemblances superficielles) entre les signes autour de lui. Il s'agit d'une tendance interprétative tous azimut. Certains neuroscientifiques (Kapur\&Mamo [42], Panksepp [44], Stahl [37]) ont corrélé ce processus à une hyperactivité de certaines structures cérébrales dopaminergiques du système mésolimbique. L'autre processus humoral serait ce que la tradition psychiatrique [52] appelle athymhormie. Celle-ci consiste en une désaffectation par rapport à l'état précédent - de l'envahissement par les signes. C'est à dire que le sujet « apathique » n'y prête plus attention, il en est désensibilisé. Selon Stahl [37] ceci se passerait, peut-être, par un déficit en dopamine au niveau des aires de projections mésocorticales et ce phénomène pourrait être en lien avec l'hyperactivité excitotoxique des systèmes glutamatergiques. Pourtant, comme nous avons vu, Stahl [37] mais aussi d'autres auteurs ( par exemple Jouvent \& Carton [53] et Luauté

${ }^{6} \mathrm{Cf}$. sur la distinction entre suppléances réelles, symboliques et imaginaires de Christian Hoffmann [50] et aussi quant à la distinction entre suppléances et compensations, l'article de Frédéric Pellion [51]. 
\& Saladini [54] avec leur lecture transnosographique de l'émoussement affectif ou de l'athymhormie), même en tirant des conclusions intéressantes pour la recherche psychopharmacologique, risquent l'assimilation des maladies neurologiques et psychiatriques.

Nous pouvons décrire des processus psychosomatiques similaires dans le cadre de la dépression, la psychose maniacodépressive, les états de panique, la névrose traumatique, les addictions et ailleurs. Nous n'avons pas pu, dans le cadre de cet article, entrer dans les détails de tous ces processus et nous nous sommes limités ainsi à montrer une voie de recherche qui va dans ce sens par le biais de la schizophrénie.

\section{Quelques implications théoriques et pratiques}

Nous allons par la suite juste indiquer de manière liminaire l'intérêt du point de vue que nous proposons pour la délimitation de la discipline de la psychiatrie par rapport à la neurologie, mais aussi pour la thérapeutique et pour la recherche à venir.

- Nous pensons avoir suffisamment indiquer de quelle manière ce type de changement sur le plan du fonctionnement, voire de la structure cérébrale, est différent dans les cas neurologiques par rapport à certains types psychopathologiques, que Stahl et d'autres auteurs, comme nous venons de voir, mettent au même niveau neuropsychiatrique. Dans le cas de certaines maladies psychiatriques, nous avons affaire avec une participation psychosomatique du cerveau à travers une réduction sémiotique qui touche électivement certains circuits cérébraux, lesquels 
ont un rapport spécial avec des opérations qui relèvent de la tiercéité sémiotique. Alors que ce n'est pas le cas pour les maladies neurologiques.

- La conclusion sur le plan de la thérapeutique de ce qui précède pourrait être, que même si, à notre avis, on peut légitimement supposer que les psychotropes agissent plutôt au niveau des automatismes de stimuli tandis que les thérapies cognitivo-comportementales agissent préférablement au niveau des associations signalétiques, ce n'est pas à ce niveau que nous pouvons escompter une guérison, du moins une guérison durable qui inclurait les capacités créatives du sujet. Car, dans la mesure où les affections psychosomatiques (au sens général que nous avons donné à ce terme) ont affaire avec la valeur suggestive des signifiants gelés, les thérapies biologiques et cognitivo-comportementales, à travers la suggestion que peuvent impliquer, elles entretiennent la réduction sémiotique et déresponsabilisent le sujet. Dans ce sens, ces thérapies sont des contre-suggestions, et leur utilité, du coup, ne serait que pour des cas qui sont, ponctuellement ou durablement, réfractaires à la mise en place des dispositifs «tiercéisants » et surtout comme moyens d'attente, afin de créer des conditions propices à l'instauration de tels dispositifs. Selon la phrase freudienne il faut parfois mélanger l'or pur de la cure psychanalytique avec le cuivre de la suggestion. C'est la cure psychanalytique qui peut surtout mobiliser, par le «capitonnage» des signifiants qu'elle produit (qui est une opération hautement « tiercéisante »), les forces créatives propres à chaque sujet.

- Le constat de certaines compatibilités de modèles entre les neurosciences et la psychanalyse comme celles que nous venons de constater à propos de 
la schizophrénie n’est pas sans conséquences pour la recherche. Dans l'exemple de la schizophrénie, nous pensons que la conceptualisation que nous proposons pourrait aider les psychiatres biologiques à réfléchir sur la manière selon laquelle s'articulent entre eux ce qu'ils appellent symptômes positifs, négatifs et cognitifs de la schizophrénie. A travers cette conceptualisation les psychanalystes sauraient-ils mieux situer la valeur de certains traitements médicamenteux et le moment opportun pour conseiller un recours à d'autres modalités thérapeutiques en général ?

\section{Conclusion}

En guise de conclusion, il ne nous paraît pas étonnant que lorsque la chaîne signifiante est mise à mal, le corps de l'être parlant commence à être envahi par cette autre logique de son organisme animale, qui est celle de signes, et qui peut le rendre malade sur un mode psychosomatique. Car la tiercéité des structures symboliques reste constitutive pour l'être parlant et la secondéité ou la priméité des systèmes signalétiques éthologiques, quand ils tendent à fonctionner indépendamment de cette tiercéité symbolique, sont nocifs pour l'organisme humain suivant des modalités psychosomatiques bien précises. Et, à trop vouloir guérir ces maladies psychosomatiques sans prendre en compte la logique signifiante, on prive le sujet de ressources qui lui sont propres, aussi bien par rapport à sa possibilité créative que par rapport à son auto-guérison - que celle-ci soit apportée par la voie de la parole dans la cure et/ou par la voie du réel du corps. 


\section{BIBLIOGRAPHIE}

1. Vanier A. Questions de symptôme. L'Évolution psychiatrique. 2001 ; 66 : 263-271

2. Vidal F. Le sujet cérébral : Une esquisse historique et conceptuelle. Psychiatrie, Sciences humaines, Neurosciences. janvier-février 2005 ; III : 37-48

3. Ehrenberg A.. Le sujet cérébral. revue Esprit. 2004 ; (nov) : 130-155

4. Price B H, Adams D, Coyle J T. Neurology and psychiatry: closing the Great Divide. Neurology $2000 ; 54: 8-14$

5. Yudofsky S T, Hales R E. Neuropsychiatry and the future of Psychiatry and Neurology. Am J Psychiatry. $2002 ; 159$ : 1261-1264

6. Thibierge S. Clinique de l'identité. Paris : PUF ; 2007

7. Ey H, Ajuriaguerra J, Hécaen H. Neurologie et psychiatrie. nouvelle édition, Paris : Hermann ; 1998

8. Lacan J. Propos sur la causalité psychique. In : Ecrits, Paris : Seuil ; 1966. p.151193

9. Lacan J. D'une question préliminaire à tout traitement possible de la psychose. In : Ecrits, Paris : Seuil ; 1966. p.531-583

10. Arieti S. «Schizophrenia ». In : Arieti S, editor. American Handbook of Psychiatry. New York : Basic Books INC Publisher; 1959. p.454-507

11. Jung C G. Psychologie de la démence précoce: essai. In : Psychogenèse des maladies mentales. Paris : Albin Michel ; 2001. p.69-187

12. Arieti S. Interpretation of schizophrenia. New York : Basic Books ; 1974

13. Dejours C, Abdoucheli E. L'interprétation psychosomatique de la schizophrénie et l'hypothèse de la somatisation cérébrale. Entrevues. 1990 (nº spécial) ; 17 : 34-45

14. Cacciali P, Froissart F. Interview de Marie-Christine Laznik. Journal Français de Psychiatrie. Autismes, controverses, perspectives thérapeutiques, $2^{\text {ème }}$ trimestre $2006 ; 25: 45-51$

15. Mentzos S. Die Neurobiologie der Psychosen - Die Psychosomatosen des Gehirns in Lehrbuch der Psychodynamik, [Les psychosomatoses du cerveau in Traité de psychodynamiques]. Göttingen : Vandenhoeck \& Ruprecht ; 2010. p.235-247

16. Pally R. The Mind-Brain Relationship. London : Karnac ; 2000. p.88-89 
17. Widlöcher D. Clinique psychanalytique et psychotropes, Dépression et anxiété. Revue Française de Psychanalyse. 2002 ; t. LXVI, Les psychotropes sur le divan: 409-422.

18. Ali S. Penser le somatique. Paris: Dunod ; 1987

19. Hartocollis P. 'Actual Neurosis' and psychosomatic Medicine: The Vicissitudes of an enigmatic Concept. International Journal of Psychoanalysis. $2002 ; 83$ :13611373

20. Verhaeghe P. On being normal and other disorders. New York : Other Press ; 2004

21. Lacan J. Le Séminaire livre XI, Les quatre concepts fondamentaux de la psychanalyse, texte établit par Jacques-Alain Miller. Paris : Seuil ; 1973

22. Lacan J. Conférence à Genève sur « Le symptôme» du 4 octobre 1975. Le blocnotes de la psychanalyse, $1985 ; 5: 5-23$

23. Auteur. Psychogenèse et organogenèse en psychopathologie [Thèse de recherches en psychanalyse]. Une hypothèse d'affection ou participation psychosomatique du cerveau. Paris : Paris-Diderot ; 2011

24. Peirce Ch S. Ecrits sur le signe: rassemblés, traduits et commentés par Gérard Deledalle. Paris : Seuil ; 1978

25. Vidal J-M. La tiercéité symbolique, fondement de la discontinuité psychique entre animaux et humains. Revue Francaise de Psychanalyse, 2011 ; (75) : 17-51

26. Lacan J. Fonction et champs de la parole et du langage. In : Écrits. Paris : Seuil ;1966. p.237-322

27. Balat M. Psychanalyse, logique, éveil de coma : Le Musement du scribe. Paris : L'Harmattan ; 2000, (coll. « ouverture philosophique »)

28. Delion P. L'enfant autiste le bébé et la sémiotique. $1^{\text {ère }}$ éd., Paris : PUF ; 2000, (coll. « Le fil rouge »).

29. Muller J. Hierarchical Models in Semiotics and Psychoanalysis. In : Muller J, Brent J, editors. Psychoanalysis, Psychiatry and the Humanities, 2000, vol.15, Peirce, Semiotics and Psychoanalysis. p.49-67

30. Roulot D. Secondéité pure et univers schizophrénique. In : Balat M,DeledallesRhodes J, Deledalles G, editors. Signs of humanity, vol II. Berlin : Mouton de Gryter ; 1992. p.1087-1096

31. Ansermet F. Magistretti P. «Quel inconscient ? ».In Magistretti P. Ansermet F., (eds). Neurosciences et Psychanalyse, Paris : Odile Jacob ; 2010. p.195-199

32. Lyons-Ruth, K. I sense that you sense that I sense...: Sander's recognition process and implicit relational moves in the psychotherapeutic setting. Infant Mental Health Journal, $2000 ; 21: 85-98$.

33. Czermak M. Patronymies. Paris : Masson ; 1998

34. Lanteri-Laura G. La notion de l'automatisme dans la médicine et dans la psychiatrie moderne. In: Grivois $\mathrm{H}$, director. Autonomie et automatisme dans la psychose. Paris : Masson ; 1992. p. 7-29 
35. Melman C. La nouvelle économie psychique. Paris : ères ; 2009

36. Post R. Transduction of Psychosocial Stress Into the Neurobiology of Recurrent, Affective Disorder. Am J Psychiatry August 1992 ; 149 8 : 999-1010

37. Stahl S. Psychopharmacologie essentielle. Paris : Flammarion ; 2002

38. Auteur. Lecture croisée de recherche en psychanalyse et en psychiatrie biologique en psychopathologie par la voie de l'automatisme. Synapse, 2005; (213) : 19-30

39. Auteur. Existe-t-il des affections psychosmatiques du cerveau? Recherche en psychanalyse [en ligne], 2009; 7, Psychanalyse, psychopathologie cognitive et neurosciences: quel débat? Disponible sur : www.recherchespsychanalyse.revues.org/?

40. Fink B. Fundamentals of psychoanalytic technique: A lacanian approach for practitioners. New York: W.W.Norton; 2007

41. Hemsley D. «A Simple (or Simplistic?) Cognitive Model for Schizophrenia ». Behaviour Research and Therapy. 1993 ; 31 : 633-646

42. Kapur S, Mamo D. Why antipsychotics are anti-'psychotic'. In : Mcdonald C, Schulze K, Murray R, Wright P, editors. Schizophrenia: challenging the orthodox. London : Taylor and Francis ; 2004. p.113-125

43. Lambert P. Psychanalyse et psychopharmacologie. Paris : Masson ; 1990

44.Panksepp J. Affective Neuroscience, The foundation of human and animal emotions. Oxford : Oxford University Press ; 1998

45. Van Os J. A salience dysregulation syndrome. Br J Psychiatry 2009 ; 194 : 101103

46. Vergotte A. Le plaisir destructeur transfiguré en hiérogamie. In : Deveresse J, Lothan Z, Shotte J. Schreber revisité. Louvain : Presses Universitaires de Louvain ; 1998. p.223-243

47. Widlöcher D. La psyché carrefour. Paris : Eshel ; 1997

48. Lacan J. De la psychose paranoïaque dans ses rapports avec la personnalité. Paris : Seuil ; 1975

49. Freud S. Le président Schreber. In : Cinq psychanalyses, $18^{\text {ème }}$ éd. Paris : PUF ; 1993. p.263-324

50. Hoffmann C. Le paradigme des suppléances psychotiques. Recherche en psychanalyse [en ligne], 2009; 7, Psychanalyse, psychopathologie cognitive et neurosciences: quel débat? Disponible sur: www.recherchespsychanalyse.revues.org/?

51. Péllion F. Quelques réflexions sur la pertinence clinique et psychopathologique de la notion de «suppléance ». Recherche en psychanalyse [en ligne], 2009; 7, Psychanalyse, psychopathologie cognitive et neurosciences : quel débat? Disponible sur : www.recherchespsychanalyse.revues.org/?

52. Guiraud P. Psychiatrie Générale. Paris : Le François ; 1950 
53. Jouvet R. Carton S. L'émotion dérégulée. In : Widlöcher D, directeur. Traité de psychopathologie. Paris : PUF ; 1994. p.561-581

54. Luauté JP, Saladini O. Le concept français d'athymhormie de 1922 à nos jours. Rev can psychiatrie. septembre $2001 ; 46: 639-643$ 